\title{
The role of the Association of British Clinical Diabetologists
}

\author{
Peter H Winocour
}

The Association of British Clinical Diabetologists (ABCD) is a young specialist society founded in 1997 as a professional organisation of consultants and latterly trainees specialising in diabetes across the UK. The primary principal for its inception was to support and promote specialist diabetes services in all settings, thereby ensuring high quality care for all living with diabetes in the UK. The impetus was in part through the changing role of diabetes service provision and the recognition that specialist diabetologists have particular responsibilities and roles that extend beyond clinical diabetes - notably their input to acute and general medicine and endocrinology. Subsequent to its establishment a new charity, the ABCD Diabetes Care Trust, was also created with the purpose of promoting best practice and education in the provision of specialist services for people with diabetes. ABCD is now securely established with over $50 \%$ of NHS consultant diabetologists and over 40\% (and growing) of higher trainees as members.

Its main achievements to date have been with collaborative surveys on specialist diabetes services and retinal screening, demonstrating continued regional disparities in service provision, and a disconnection between the process and holistic diabetes-opthalmology care after detection of important retinopathy. A national diabetes audit programme has been established covering areas such as acute coronary syndrome glycaemic care, out-of-hospital hypoglycaemia management, inpatient glycaemic care and independent assessment of new injectable glucagon-like peptide-1 analogues.

Dialogue and discussion seem to be the best mechanism for improving services and spreading best practice and twice-yearly national meetings serve an important role in enabling interactive discussion. They are structured to enable mature discussion of controversial topics in an atmosphere where all views from all levels are encouraged. Meetings are designed to maximise continuing professional development opportunities.

$\mathrm{ABCD}$ is a key stakeholder as a professional organisation inputting to the National Institute for Health and Clinical Excellence (NICE) technology and guidelines programmes. Its role in education has been strengthened through input to the Specialty Certificate Examination (SCE) in diabetes and endocrinology and the speciality training committee (STC), and sponsorship of a King's Fund leadership course for training registrars in diabetes and endocrinology. It has worked closely with other organisations, notably the Royal College of Physicians (RCP) with whom a successful meeting on inpatient diabetes

Peter H Winocour, chairman, Association of British Clinical Diabetologists care has been organised with whom Diabetes Teams without Walls was developed as an exemplar for integrated care. The ABCD also inputs to the RCP Council focusing on specialty-acute general internal medicine interface issues.

The diabetes community works best together - the ABCD is in close collaboration with Diabetes UK (the national patient charity) and the Primary Care Diabetes Society. The next version of the Joint British Societies' Guidelines on Prevention of Cardiovascular Disease (JBS 3) will have ABCD input enabling an update of the role of aspirin in primary prevention and the role of statins in type 1 diabetes. Position statements on new therapies are designed to offer practical if challenging enhancements of NICE guidance.

ABCD was instrumental in establishing the Joint British Diabetes Societies (JBDS) inpatient working group. This was in recognition of the high priority afforded this area of practice in the National Service Framework for Diabetes and confirmed by the recent National Diabetes Inpatient Audit which stated that up to $20 \%$ of inpatients in England had comorbid diabetes or a primary admission through diabetes. The quality of inpatient diabetes care has been found to be extremely variable, leading to increased length of stay, adverse (sometimes fatal) outcomes from acute illness, poor quality patient experiences and increased readmission rates. The publication of contemporary practical guidelines for managing diabetes metabolic emergencies by the JBDS is designed to rectify these anomalies along with a strong push from ABCD to ensure that every acute hospital has a dedicated diabetes inpatient service led by a consultant diabetologist.

Of course as a chronic disease it is just as vital that there is supported self-management and that specialist diabetes care is provided in line with the Darzi White Paper Closer to home where appropriate. ABCD are pushing the mantra of ensuring that people living with diabetes are seen at 'the right place, at the right time, by the right person', and fully support the core statement from the national clinical director for diabetes to ensure 'prompt expert advice' to deal with problems. This will require important expansion of diabetes consultant posts, and a more varied approach to job planning. This is likely to be a model for many other areas of specialist medical practice in the future.

One of ABCD's major roles has been the understanding of the current and changing role of the physician with an interest in diabetes and endocrinology in the district general hospital setting and how this can best change with the dynamics of acute medicine, hospital wide inpatient diabetes care, service reconfigurations and the creation of foundation trusts, and integrated diabetes care, with specialists providing support to primary care colleagues. 
ABCD has been fortunate to meet with colleagues from more established specialist societies from whom the organisation has learnt a great deal about advancing key objectives working closely alongside the RCP, especially in rolling out specialist revalidation. Successful diabetes care is all about teamwork. ABCD will take these principles forward in working with the RCP, other medical specialist societies and, vitally, colleagues in community and primary care.

The next decade of financial austerity within the NHS will compel us all to have a more imaginative and sometimes radical approach to how best to bring our skills as physicians into different areas of practice. However, in line with many other organisations,
ABCD supports the view that cost- and clinically-effective care is best served within the NHS, minimising the risk of fragmented care, which is clearly inappropriate for the increasing number of people living with a variety of 'chronic diseases'.

Address for correspondence: Dr PH Winocour,

Department of Diabetes and Endocrinology,

Queen Elizabeth II Hospital,

Welwyn Garden City, Herts AL7 4HQ.

Email: peter.winocour@nhs.net

\title{
RCP BOOKS
}

\section{Hoffenberg: Physician and humanitarian}

\author{
by L Ross Humphreys
}

Born in South Africa in 1923 - where he trained and practised as a physician and from which he was banned for his anti-apartheid activities in 1966 - Raymond (Bill) Hoffenberg was to become a familiar and highly respected figure in the worlds of academe and medicine in the UK. He became president of both the Royal College of Physicians of London and Wolfson College, Oxford posts which for a time he held simultaneously.

This well researched biography charts Hoffenberg's life from early childhood in Port Elizabeth. It includes a revealing account of the time he served as a stretcher bearer in the South African army (which he joined when under age by forging his father's signature) through to his medical research career at Groote Schuur and his chairmanship of the Defence Aid Fund that financed the defence of people accused of political crimes in South Africa.

As a young physician in South Africa in 1967, he was asked to remove a still beating heart for transplant to one of Christian Barnard's patients - an experience that led him to pursue clear criteria for the clinical diagnosis of death. This, along with end of life issues, and the availability of organs for transplant were all issues pursued by Hoffenberg through the organisations that he headed or to which he was affiliated; these issues remain high on the medical, public and government agendas today. A powerful, tall physique allowed him to excel in many sports in his younger days, whilst a towering intellect coupled with organisational flair, tenacity and charm enabled Hoffenberg to rise

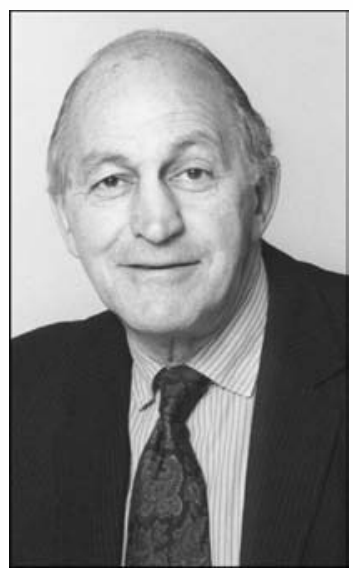
to high office. But the characteristics for which he will best be remembered by his colleagues and friends were his compassion, a gift for friendship and his prodigious capacity for enjoyment which enhanced the lives of all who knew him.

\section{Contents}

- Port Elizabeth - A lance corporal in North Africa and Italy

- A physician's focus on research - Human rights in South

Africa - Hoffenberg banned - Birmingham - Wolfson College, Oxford $\bullet$ Maintaining standards of medical practice

- The delivery of health services - Medical ethics

- Medical activist • The later years

Published January 2010 ISBN 9781860163661

Price: $£ 20.00$ UK, $£ 22.00$ overseas (prices include postage and packing)

$10 \%$ discount on all RCP publications to fellows and members

Please quote the reference Clinical Medicine when making your order

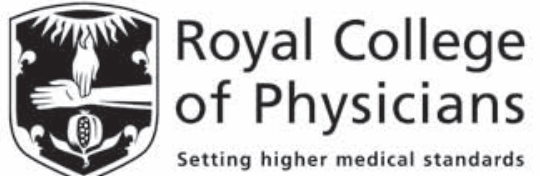

